\title{
Au-poly(lactic-co-glycolic) acid complex nanoparticles as ultrasound contrast agents: Preparation, characterization and in vitro study
}

\author{
XIAO-YU LI* ${ }^{*}$, LI XU* ${ }^{*}$, HONG-LI LI, JING DU, XUE-SONG LIU and FENG-HUA LI \\ Department of Ultrasound, Renji Hospital, School of Medicine, Shanghai Jiao Tong University, Shanghai 200127, P.R. China
}

Received January 24, 2016; Accepted February 10, 2017

DOI: $10.3892 / \mathrm{mmr} .2017 .8351$

\begin{abstract}
The present study aimed to prepare and characterize an Au-poly(lactic-co-glycolic) acid (PLGA) complex, surfaced with gold nanoparticles (GNPs), and to assess its use as an ultrasound contrast agent for in vitro imaging. PLGA nanoparticles (NPs) were prepared by a modified double-emulsion solvent evaporation method. GNPs were prepared via reduction with sodium citrate. Au-PLGA complexes were created through the binding of polyethylene glycosylated GNPs to the surface of PLGA NPs. Various physical characteristics of PLGA NPs, GNPs and Au-PLGA complexes, including morphology, size, dispersion, $\zeta$ potential and stability were observed and measured. The study of the in vitro imaging capability of the Au-PLGA complex was conducted by setting multi-parameter imaging conditions. The average sizes of PLGA NPs, GNPs and the Au-PLGA complex were 144.5 $\pm 30.28,21.42 \pm 1.56$ and $359.4 \pm 67.94 \mathrm{~nm}$, respectively. Transmission electron microscopy (TEM) revealed that PLGA NPs and GNPs were spherically shaped, but GNPs had smooth surface while PLGA NPs had a capsular morphology with a wrinkled surface and large voids. The maximum absorption peak of $20 \mathrm{~nm}$ GNPs was $508 \mathrm{~nm}$ while that of the Au-PLGA complex was $515 \mathrm{~nm}$ on an ultraviolet-visible absorption spectrum curve. The results of TEM and the redshift spectrum demonstrated that GNPs successfully bound to the surface of the PLGA NPs. The complex demonstrated good stability and its size changed little over time. In vitro imaging experiments revealed that the Au-PLGA complex had a good imaging ability. The present study demonstrated that the Au-PLGA complex is a promising novel ultrasound contrast agent.
\end{abstract}

Correspondence to: Professor Feng-Hua Li, Department of Ultrasound, Renji Hospital, School of Medicine, Shanghai Jiao Tong University, 1630 Dongfang Road, Shanghai 200127, P.R. China E-mail: fenghua-li@163.com

*Contributed equally

Key words: gold nanoparticles, poly(lactic-co-glycolic acid) nanoparticles, ultrasound contrast agents

\section{Introduction}

Gold nanoparticles (GNPs) have good optical properties, good biocompatibility and are harmless. Therefore, GNPs have been applied to biochemical and medical analysis, biosensors, genomics, immunology, wound healing, and tumor diagnosis and treatment (1). The nano-gold complex consists of gold nanoparticles with a diameter of 1-100 nm (2) surface-modified by covalent or non-covalent bonding to specific molecules or drugs, which are used in the diagnosis and treatment of multiple cancers. GNPs exhibit enormous potential, from basic research to clinical applications. Its use as a treatment for cancer has been approved by the FDA and has entered the early stages of clinical trials (3). The surface of GNPs readily binds to chemotherapy drugs and functional ligands (4). This combination of capabilities extends the functionality and applications of GNPs, and they have also been used as molecular probes, and for optical imaging. Song et al (5) used gold nanorods as lymph tracer in a mouse model and successfully obtained sentinel lymph node photoacoustic imaging in vivo.

Polymer microbubbles are an ultrasound contrast agent that demonstrates good uniformity, high stability, a long retention time in vivo, good anti-stress performance and weak sound attenuation characteristics. Currently the most widely used is poly (lactic-co-glycolic) acid (PLGA). Néstor et al (6) evaluated the echogenic power and stability of air-filled PLGA nanocapsules with a mean diameter of $370 \pm 96 \mathrm{~nm}$ in vitro. Kohl et al (7) prepared and evaluated multifunctional PLGA nanoparticles (NPs) for use in photoacoustic imaging. $\mathrm{Xu}$ et al (8) developed PLGA nanobubbles (mean diameter $268 \mathrm{~nm}$ ) for cancer targeting and imaging. Ke et al (9) prepared the nanocomposite through combined GNPs with a polymer microcapsule shell to operate as a new multi-functional agent for both contrast enhanced ultrasonic imaging and photohyperthermia. Good ultrasonic imaging results were achieved as a result.

Therefore, it is possible to hypothesize that the combination of gold and polymer nanobubbles significantly increases the echo signal of the PLGA nanobubble contrast agent, improving the ultrasound contrast performance. The aim of the present study was to prepare a gold-poly(lactic-co-glycolic) acid (Au-PLGA) complex and investigate the feasibility of using this complex to enhance ultrasound imaging in vitro. 


\section{Materials and methods}

Materials. PLGA (50:50; 40k MW) was obtained from Shandong Jinan Daigang Biomaterial Co., Ltd. (Shandong, China). Thiol-terminated methoxy polyethylene glycol $\left(\mathrm{H}_{2} \mathrm{~N}-\mathrm{PEG} 1000-\mathrm{SH} ; 1 \mathrm{k} \mathrm{MW}\right)$ was obtained from Shanghai Yare Biotech, Co., Ltd. (Shanghai, China). Methylene chloride, isopropanol, mannitol, phosphotungstic acid, hydrogen tetra-chloroaurate (III) hydrate $\left(\mathrm{HAuCl}_{4} \bullet 4 \mathrm{H}_{2} \mathrm{O}\right)$ and sodium citrate were obtained from Sinopharm Chemical Reagent Co., Ltd. (Shanghai, China). Polyvinyl alcohol (PVA; $88 \%$ mole hydrolyzed), camphor (D+), 1-(3-Dimethylaminopropyl)-3-ethylcarbodiimide hydrochloride and N-Hydroxysuccinimide (NHS) were obtained from Aladdin Chemistry Co., Ltd. (Shanghai, China). Deionized (DI) water, used in all experiments, was purified using a Milli-Q Plus 185 water purification system (EMD Millipore, Billerica, MA, USA) with resistivity $>18 \mathrm{M} \Omega \mathrm{cm}$. All other chemicals and reagents were of analytical grade.

Preparation of PLGA NPs. PLGA NPs were prepared according to a previously reported method (10). To make the first emulsion, PLGA $(125 \mathrm{mg})$ and camphor $(12.5 \mathrm{mg})$ were dissolved in methylene chloride $(5 \mathrm{ml})$, followed by adding $1 \mathrm{ml}$ PVA (3\% w/v). Then, the mixture was emulsified using an ultrasonic disrupter 04717 (Cole-Parmer Instrument Co., Ltd., Vernon Hills, IL, USA) at $130 \mathrm{~W}$ in an ice bath and sonicated for $180 \mathrm{sec}$ at $4 \mathrm{sec}$ on and $2 \mathrm{sec}$ off. To make the second emulsion, the solution was added to $20 \mathrm{ml}$ PVA $(3 \% \mathrm{w} / \mathrm{v})$ and sonicated as above. Following this, the emulsion was added to $100 \mathrm{ml}$ isopropyl alcohol $(5 \% \mathrm{v} / \mathrm{v})$ and stirred for $1 \mathrm{~h}$. The samples were subsequently obtained by centrifugation at $17,800 \mathrm{xg}$ for $10 \mathrm{~min}$ at $25^{\circ} \mathrm{C}$, washed three times with DI water, and lyophilized using a freeze dryer (TFD5505; Ilshin Lab Co., Ltd., Yangju, South Korea). In a vacuum, the PLGA NPs was filled with perfluoropropane gas (Shanghai Renjieling Optics Instruments Co., Ltd., Shanghai, China).

Preparation of GNPs. GNPs with an average diameter of $\sim 20 \mathrm{~nm}$ were prepared according to the previously reported method $(11,12)$. $\mathrm{HAuCl}_{4} \bullet 4 \mathrm{H}_{2} \mathrm{O}$ aqueous solution $(0.5 \mathrm{ml}$; $1 \% \mathrm{w} / \mathrm{v}$ ) was added into $48 \mathrm{ml}$ DI water, and the solution was stirred and heated in an oil bath so that the temperature was raised and stabilized at $137^{\circ} \mathrm{C}$. Under vigorous stirring, $1.5 \mathrm{ml}$ sodium citrate solution $(1 \% \mathrm{w} / \mathrm{v})$ was added, and stirring continued for 20 min until the solution became a clear red. It was subsequently removed from the oil bath and cooled to room temperature with constant stirring.

The GNP solution was prepared by filtration through a $0.22 \mu \mathrm{m}$ nitrocellulose membrane, so as to remove electrolytic ions and aggregated colloidal gold particles. Glassware used for the experiment was subjected to several rinses with DI water and acidification.

Preparation of Au-PLGA complex. $\mathrm{H}_{2} \mathrm{~N}-\mathrm{PEG1000- \textrm {SH }}(30 \mathrm{mg})$ was dissolved in $10 \mathrm{ml}$ filtered GNP solution and continuously stirred at room temperature for $4 \mathrm{~h}$. PLGA NPs $(30 \mathrm{mg})$ was fully dissolved in DI water and kept standing for $20 \mathrm{~min}$ to discard large particles, which precipitated at the bottom. The suspension was centrifuged at $17,800 \times g$ for $10 \mathrm{~min}$ at $25^{\circ} \mathrm{C}$ and washed with DI water three times to obtain purified PLGA NPs. Subsequently, the PLGA NPs were mixed with EDC $(3 \mathrm{mg})$ and NHS $(2 \mathrm{mg})$ in $10 \mathrm{ml}$ DI water. The resulting solution was stirred at room temperature for $2 \mathrm{~h}$, then centrifuged at $17,800 \mathrm{xg}$ for $10 \mathrm{~min}$ at $25^{\circ} \mathrm{C}$ and washed with DI water to expose the carboxyl groups (-COOH) of PLGA NPs. Then, the PLGA NPs were completely suspended in GNP solution and stirred at room temperature for another $2 \mathrm{~h}$ to obtain the Au-PLGA complex.

Characterization of PLGA NPs, GNPs and the Au-PLGA complexes. The S-4800 field emission scanning electron microscope (Hitachi High Technologies Corporation, Tokyo, Japan) and JEOL JEM-2100 low to high-resolution transmission electron microscope (TEM; Hitachi High Technologies Corporation, Tokyo, Japan) were used to observe the morphology and structure of the nanoparticles. The PLGA NPs were stained with freshly prepared phosphotungstic acid solution $(1 \%, w / v)$ prior to TEM observation. A drop of the PLGA NPs suspension was placed onto copper grids for $3 \mathrm{~min}$ and then the copper grids was immersed in phosphotungstic acid solution for $1 \mathrm{~min}$. The grids were air-dried and observed by TEM. The size distributions and $\zeta$ potentials of the nanoparticles were evaluated five times for each sample using dynamic light scattering (Zetasizer Nano ZS model ZEN3690; Malvern Instruments, Ltd., Malvern, UK). The Varian 4000 UV-Vis spectrophotometer was used to measure the ultraviolet (UV)/Vis absorption spectra of the nanoparticles. To investigate the preserved stability of the Au-PLGA complex, sample suspensions were sufficiently ultrasonically dispersed using the mixing function of an ultrasonic cleaning machine set to $500 \mathrm{~W}$ and kept standing for $0,1,7$ and 14 days at $4^{\circ} \mathrm{C}$. They were subsequently measured five times for each sample to obtain the average diameters and $\zeta$ potentials.

In vitro ultrasound imaging of the Au-PLGA complex. Au-PLGA complexes and PLGA NPs were dispersed in the degassed deionized water, and different imaging parameters (imaging times 0, 30, 60, 120 and $180 \mathrm{sec}$ ) and conditions (concentration of sample 3, 2, 1, 0.5 and $0.25 \mathrm{mg} / \mathrm{ml}$ ) were selected according the purpose of the different experiments in order to observe the corresponding imaging ability. The imaging evaluation of the Au-PLGA complexes and PLGA NPs were performed using an ultrasonic diagnostic instrument (MyLab Twice; Esaote SpA, Florence, Italy) and conventional B-mode sonograms (mechanical index $=0.06$ ). The center frequencies of the transducers were 13 and $22 \mathrm{MHz}$, according to need.

Statistical analysis. The results were presented as the mean \pm standard deviation.

\section{Results}

Preparation and characterization of nanoparticles. The morphology and structure of PLGA NPs, GNPs and the Au-PLGA complexes were characterized by transmission electron microscopy (TEM). The GNPs (Fig. 1) and PLGA NPs (Fig. 2) demonstrated a spherical shape. GNPs had a more uniform size and PLGA NPs demonstrated good dispersion. 




Figure 1. Transmission electron microscopy image of gold nanoparticles.

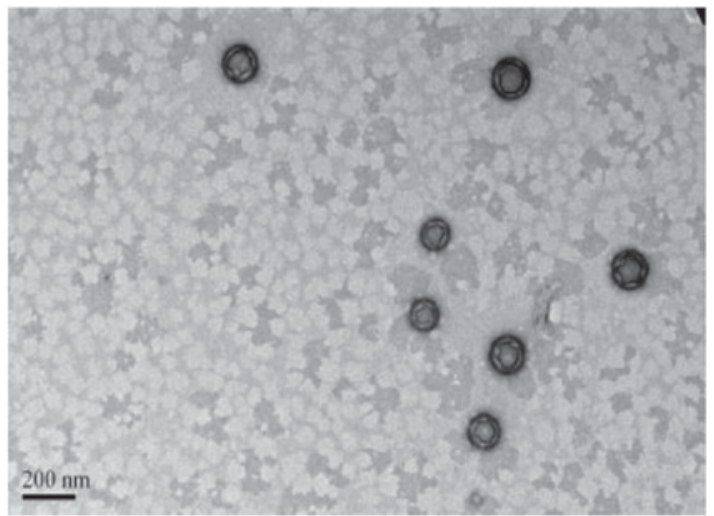

Figure 2. Transmission electron microscopy image of poly(lactic-co-glycolic) acid nanoparticles.

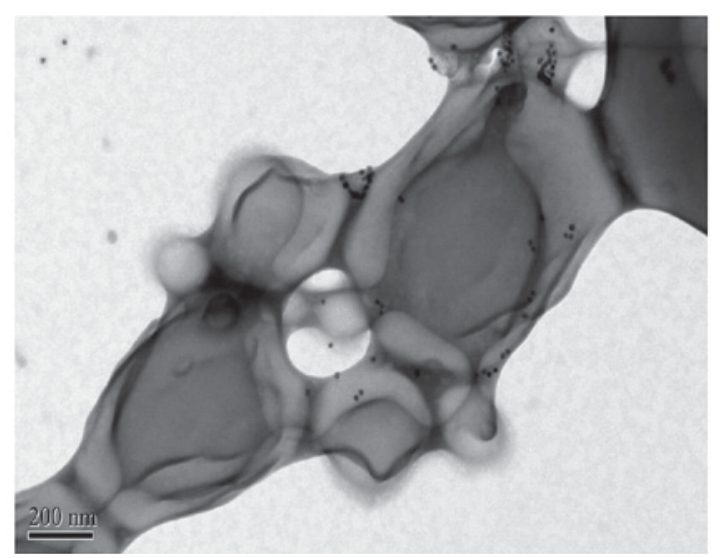

Figure 3. Transmission electron microscopy image of the goldpoly(lactic-co-glycolic) acid complex, revealing the typical capsular structure, where the polymeric shell appears darker.

TEM revealed the PLGA NPs had a thin shell that was not smooth but wrinkled, due to the TEM electron beam across the thin, cavity-rich particles (Fig. 2). DLS revealed that the GNPs and PLGA NPs had diameters of $21.42 \pm 1.56$ and $144.5 \pm 30.28 \mathrm{~nm}$, respectively (Table I). The Au-PLGA complexes also had spherical shape following the combination of GNPs with PLGA NPs (Figs. 3 and 4) and the average diameter of the complex increased to $\sim 359.4 \pm 67.94 \mathrm{~nm}$ (Table I). As time passed from 0 to 14 days, the diameters and $\zeta$ potentials of the Au-PLGA complexes changed from $359.4 \pm 67.94$ to $398.19 \pm 42.34 \mathrm{~nm}$ and
Table I. Physical characteristics of nanoparticles $(\bar{\chi} \pm S)$.

\begin{tabular}{lcc}
\hline Nanoparticles & Diameter $(\mathrm{nm})$ & $\zeta$-potential $(\mathrm{mv})$ \\
\hline GNPs & $21.42 \pm 1.56$ & $-28.8 \pm 3.88$ \\
PLGA & $144.5 \pm 30.28$ & $-25.20 \pm 4.01$ \\
Au-PLGA & $359.4 \pm 67.94$ & $-4.80 \pm 4.52$ \\
\hline
\end{tabular}

GNPs, gold nanoparticles, PLGA, poly(lactic-co-glycolic) acid.

$-4.80 \pm 4.52$ to $-4.96 \pm 3.02 \mathrm{mv}$, respectively, indicating that it is stable (Table II). UV-visible extinction spectra revealed that GNPs have a maximum absorption peak at $508 \mathrm{~nm}$, while the Au-PLGA complex had a maximum absorption peak at $515 \mathrm{~nm}$ (Fig. 5). The maximum peak absorption curve demonstrated redshift, which determined the initial PLGA NPs and GNPs combined with each other successfully.

In vitro imaging. The effect of different frequencies of imaging was studied under a certain concentration. The same concentrations $(3 \mathrm{mg} / \mathrm{ml})$ of the Au-PLGA complex and PLGA NPs ultrasound contrast agents at $22 \mathrm{MHz}$ and $13 \mathrm{MHz}$ produced good images, but at $22 \mathrm{MHz}$ demonstrated improved echoing (Fig. 6). At the same frequency under $22 \mathrm{MHz}$ or $13 \mathrm{MHz}$ condition, the echo signal of the Au-PLGA complexes was stronger than PLGA NPs.

The ultrasound signals gradually decreased with decreasing concentrations of Au-PLGA complexes and PLGA NPs at $22 \mathrm{MHz}$ (Fig. 7). The strongest echo was demonstrated at the maximum $3 \mathrm{mg} / \mathrm{ml}$ for Au-PLGA complexes and PLGA NPs. At the same concentration of Au-PLGA complexes and PLGA NPs, the signal intensity of the former was improved compared with the latter.

Furthermore, the effect of imaging time on the use of Au-PLGA complexes and PLGA NPs as ultrasound contrast agents was investigated in vitro under the conditions of $22 \mathrm{MHz}$ and the concentration of $2 \mathrm{mg} / \mathrm{ml}$. The imaging ability of the Au-PLGA complexes and PLGA NPs visibly weakened as time increased (Fig. 8). The echo signal intensity and strength of the Au-PLGA complexes were superior to the PLGA NPs across the entire imaging process, but a deposition of the Au-PLGA complex occurs at $30 \mathrm{sec}$ while PLGA NPs demonstrated a more uniform ultrasonic signal without deposition across the entire imaging process. The signals had been distributed and dissipated evenly.

\section{Discussion}

Ultrasound contrast agents alter the fundamental function of the acoustic wave (absorption, reflection and refraction), so the sites where the echo signal is enhanced have significant differences in background. Therefore, ultrasound contrast agents are used for the diagnosis and identification of heart, liver, breast and other organ diseases. Previous studies have demonstrated that the material characteristics of polymer contrast agents are superior to proteins, lipids, carbohydrates and surfactants in multiple ways $(13,14)$. Thus, polymer ultrasound contrast agents have become an important synthetic material. 


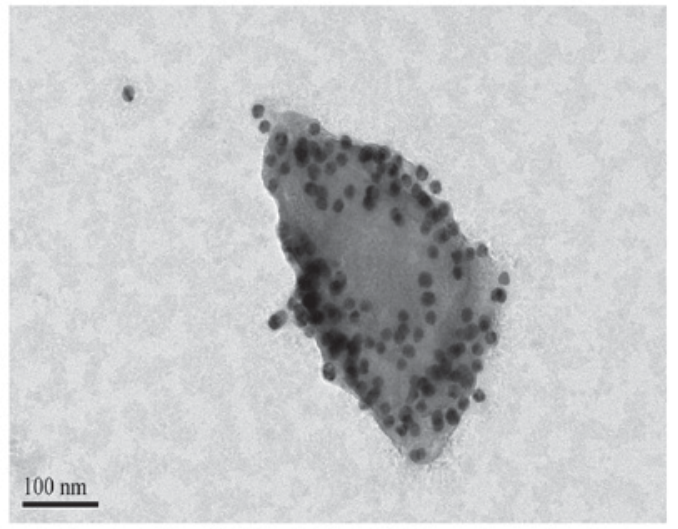

Figure 4. Transmission electron microscopy image of one goldpoly(lactic-co-glycolic) acid complex, revealing the gold nanoparticles attached to the poly(lactic-co-glycolic) acid nanoparticles.

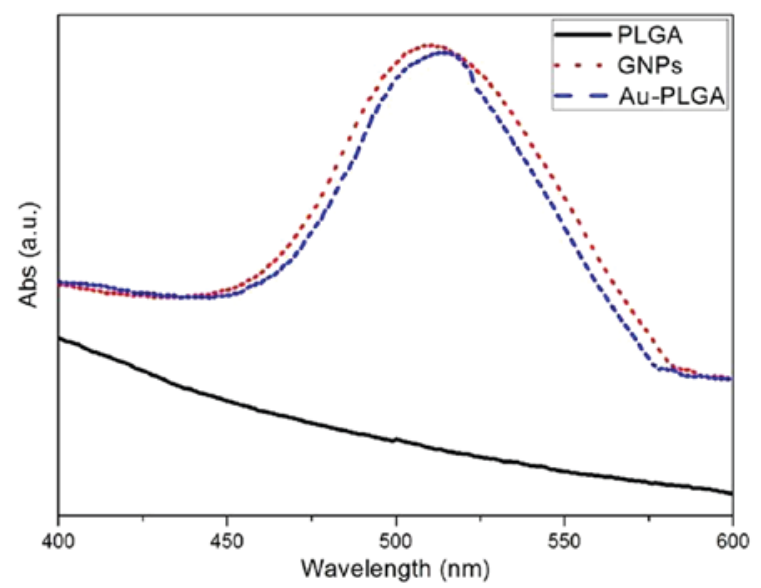

Figure 5. Ultraviolet-visible spectra of GNPs, PLGA NPs and the Au-PLGA complex. GNPs, gold nanoparticles; PLGA NPs, poly(lactic-co-glycolic) acid; NPs, nanoparticles.

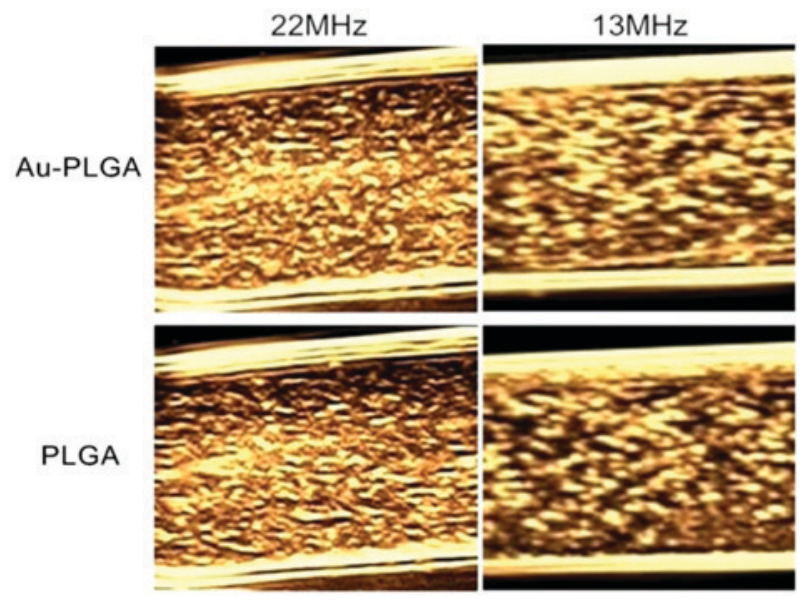

Figure 6. In vitro ultrasound images of $3 \mathrm{mg} / \mathrm{ml} \mathrm{Au-PLGA} \mathrm{complexes} \mathrm{and}$ PLGA NPs at 13 and $22 \mathrm{MHz}$. PLGA, poly(lactic-co-glycolic) acid; NPs, nanoparticles.

Preliminary results revealed that PLGA NP ultrasound imaging contrast agents, prepared with the method of an adjusted double emulsion solvent evaporation, had positive effects in in vitro and in vivo ultrasound imaging experiments (15). Therefore, the same method was adopted in the present study: A modified double-emulsion solvent evaporation method to prepare PLGA NPs with a nanobubble structure. In the formation process of polymer nanobubbles, the evaporation of the solvent and PLGA film exhalation result in the formation of polymer nanospheres that contain water. Results of previous research have demonstrated that surfactant adsorbs more readily on the gas/liquid interface when freeze drying, forming a more stable microbubble structure (16). Following freeze drying, nanospheres containing moisture and camphor were sublimed and the shape remained unchanged. Thus, an internal hollow nanocapsule was obtained. As gas molecules are able to enter the microcapsule by molecular diffusion or osmosis, an internal, hollow microbubble of gas is eventually obtained (17). The double emulsion solvent evaporation method is simple, requires mild reaction conditions, and it is possible to adjust the preparation of the micro bubble particle size, uniform and controllable particle size, shell properties and internal structure through material selection and preparation technology $(18,19)$. Thus, suitable polymer nanobubbles were obtained.

TEM images of PLGA following phosphotungstic acid dyeing revealed that it had a visible shell membrane structure, but the nanosphere surface was wrinkled. The reason for this was that PLGA NPs contain voids and the shell is thinner, so when the strong electron beam of TEM hit the particles the shell membrane retracted and formed the wrinkles. Thin shell structure permits the use of smaller amounts of ultrasonic energy that make the nanobubbles burst. This feature may be advantageous to investigate target enhancement effects in future experiments concerning targeted contrast agents in in vivo tumor models. There was a certain degree of adhesion between the Au-PLGA complexes (Fig. 3). This was again due to the TEM electron beam, which fused the borders of the polymer nanobubbles. During the experiment, it was demonstrated that when using TEM-focused photograph samples, electron microscopy produces a high-energy electron beam. This will break the shell membrane structure of polymer materials and the nanobubble will deform and fracture quickly, which is not conducive to observations. Fig. 4 presents a TEM photo of a single nanobubble complex. It is possible to observe that the complex nanosphere structure has been destroyed, and this may have been caused by a bombardment of high-energy electron beams on the nanobubble. However, more GNPs adhered to the surface of the PLGA NPs. They appeared as scattered black spots in Fig. 4, 20 nm in diameter with uniform size distribution, uniform distribution in the shell and no visible aggregation.

The oxidation of sodium citrate reduction method is the earliest method of preparation of water-soluble GNPs, and is also the most widely used. In the present study, the sodium citrate reduction method prepared $20 \mathrm{~nm}$ GNPs that demonstrated good physical and chemical properties. The UV visible absorption spectrum of GNPs revealed a maximum absorption peak of $508 \mathrm{~nm}$. When the UV spectrum of GNPs prior to and following combination with PLGA NPs, it is possible to see that the maximum peak of absorption curve demonstrated redshift. According to a previous report (20), when GNPs and the surrounding medium or matrix material interact, it may 
Table II. Physical characteristics of the Au-PLGA complex $(\bar{\chi} \pm S)$.

\begin{tabular}{lcccc}
\hline Au-PLGA & 0 days & 1 day & 7 days & 14 days \\
\hline Diameter $(\mathrm{nm})$ & $359.4 \pm 67.94$ & $365.19 \pm 32.47$ & $377.16 \pm 23.54$ & $398.19 \pm 42.34$ \\
$\zeta(\mathrm{mv})$ & $-4.80 \pm 4.52$ & $-4.57 \pm 2.32$ & $-4.6 \pm 2.7$ & $-4.96 \pm 3.02$ \\
\hline
\end{tabular}

PLGA, poly(lactic-co-glycolic) acid.

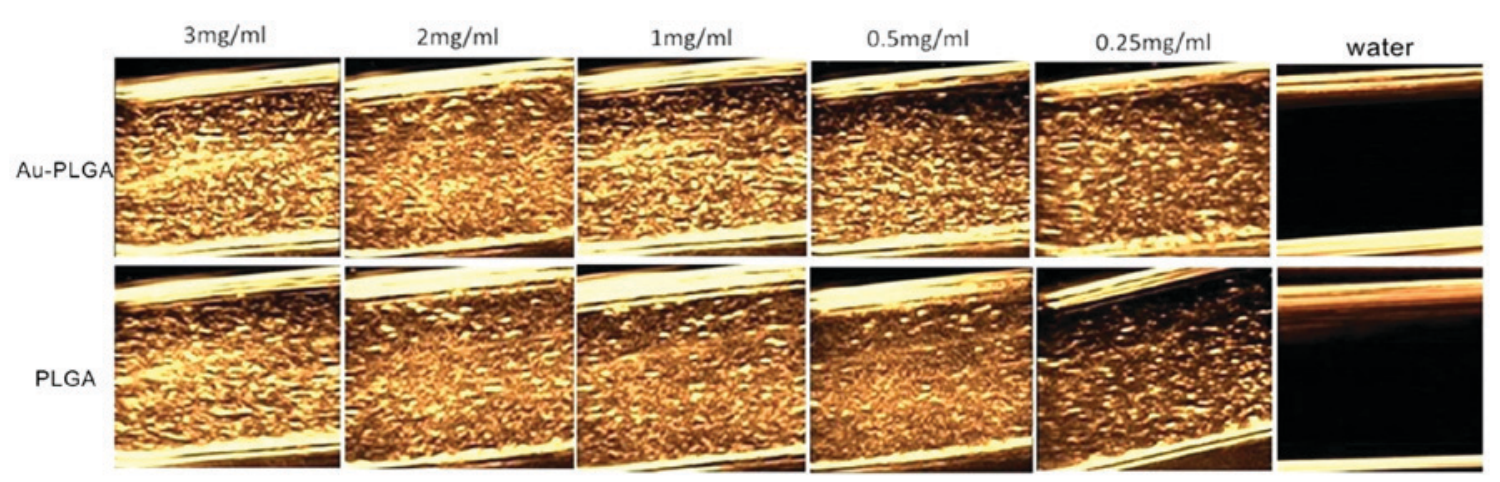

Figure 7. In vitro ultrasound images of Au-PGLA complexes and PLGA NPs at 3, 2, 1, 0.5 and $0.25 \mathrm{mg} / \mathrm{ml}$ concentrations, at $22 \mathrm{MHz}$. PLGA, poly(lactic-co-glycolic) acid; NPs, nanoparticles.



Figure 8. In vitro ultrasound images of Au-PGLA complexes and PLGA NPs (concentration $2 \mathrm{mg} / \mathrm{ml}$ ) at $22 \mathrm{MHz}$ at $0,30,60,120$ and 180 sec. PLGA, poly(lactic-co-glycolic) acid; NPs, nanoparticles.

cause absorption redshift. This determined that GNPs successfully combined with PLGA NPs due to the right shift of the maximum absorption peak. The successful combination was also demonstrated by TEM. Thiol compounds easily and firmly bind to the surface of the GNPs. Under the action of the decoupling activator NHS/EDC, the carboxylic acid group (-COOH) on the surface of the PLGA nanobubbles was activated, permitting it to combine with amino groups $\left(-\mathrm{NH}_{2}\right)$ or form other chemical bonds. In the present study, the double-ended compound $\mathrm{H}_{2} \mathrm{~N}-\mathrm{PEG} 1000-\mathrm{SH}$ was used as an intermediary to combine the thiol group with the surface of the GNPs and the amino group with the activated carboxylic acid group to form the Au-PLGA complex. This complex has good stability and the diameter of the Au-PLGA complex theoretically permits them to enter through the tumor endothelial gap (400-600 nm) (21).

Previous studies have confirmed that nanoscale ultrasound contrast agents in blood circulation possess a low echo reflection, but an accumulation of a large amount of nanoscale contrast agents significantly enhances the echo of the signal, more effectively detecting lesions $(22,23)$. In theory, the Au-PLGA complex, which includes PLGA nanobubbles containing gas and gold metal atoms, enhances the echo reflected signal more than nanobubble alone. In vitro ultrasound imaging results revealed that under conditions of high-frequency ultrasound, the ultrasound imaging ability of Au-PLGA complexes was improved compared with PLGA NPs. Under the same concentration, it has a higher echo signal strength. Compared with PLGA NPs, it deposits more readily. The reason may be the load of GNPs on the surface of PLGA NPs, which increases the quality of a single complex and results in the shortening of the suspension stability time.

In conclusion, Au-PLGA complexes were successfully constructed and the results of in vitro studies demonstrated that the Au-PLGA complexes exhibited effective imaging 
capabilities. Thus, Au-PLGA complexes hold potential as ultrasound contrast agents. However, further experimental verification in vivo is still required.

\section{Acknowledgements}

The present study was supported by the Natural Science Foundation of Shanghai (grant no. 15ZR1425600) and the National Natural Science Foundation of China (grant no. 81571678).

\section{References}

1. Khlebtsov N, Bogatyrev V, Dykman L, Khlebtsov B, Staroverov S, Shirokov A, Matora L, Khanadeev V, Pylaev T, Tsyganova N and Terentyuk G: Analytical and theranostic applications of gold nanoparticles and multifunctional nanocomposites. Theranostics 3: 167-180, 2013

2. Jain S, Hirst DG and O'sullivan JM: Gold nanoparticles as nove agents for cancer therapy. Br J Radiol 85: 101-113, 2012.

3. Kim BY, Rutka JT and Chan WC: Nanomedicine. N Engl J Med 363: 2434-2443, 2010.

4. Yeh YC, Creran B and Rotello VM: Gold nanoparticles: Preparation, properties, and applications in bionanotechnology. Nanoscale 4: 1871-1880, 2012.

5. Song KH, Kim C, Maslov K and Wang LV: Noninvasive in vivo spectroscopic nanorod-contrast photoacoustic mapping of sentinel lymph nodes. Eur J Radiol 70: 227-231, 2009.

6. Néstor MM, Kei NP, Guadalupe NA, Elisa ME, Adriana GQ and David QG: Preparation and in vitro evaluation of poly(D, L-lactide-co-glycolide) air-filled nanocapsules as a contrast agent for ultrasound imaging. Ultrasonics 51: 839-845, 2011.

7. Kohl Y, Kaiser C, Bost W, Stracke F, Fournelle M, Wischke C, Thielecke H, Lendlein A, Kratz K and Lemor R: Preparation and biological evaluation of multifunctional PLGA-nanoparticles designed for photoacoustic imaging. Nanomedicine 7: 228-237, 2011.

8. Xu JS, Huang J, Qin R, Hinkle GH, Povoski SP, Martin EW and Xu RX: Synthesizing and binding dual-mode poly(lactic-co-glycolic acid) (PLGA) nanobubbles for cancer targeting and imaging. Biomaterials 31: 1716-1722, 2010

9. Ke H, Wang J, Dai Z, Jin Y, Qu E, Xing Z, Guo C, Yue X and Liu J: Gold-nanoshelled microcapsules: A theranostic agent for ultrasound contrast imaging and photothermal therapy. Angew Chem Int Ed Engl 50: 3017-3021, 2011.

10. Xu RX, Huang J, Xu JS, Sun D, Hinkle GH, Martin EW and Povoski SP: Fabrication of indocyanine green encapsulated biodegradable microbubbles for structural and functional imaging of cancer. J Biomed Opts 14: 034020, 2009.
11. Frens G: Controlled nucleation for the regulation of the particle size in monodisperse gold suspensions. Nature 241: 20-22, 1973.

12. Horisberger $M$ and Rosset J: Colloidal gold, a useful marker for transmission and scanning electron microscopy. J Histochem Cytochem 25: 295-305, 1977.

13. Cózar-Bernal MJ, Holgado MA, Arias JL, Muñoz-Rubio I, Martín-Banderas L, Alvarez-Fuentes J and Fernández-Arévalo M: Insulin-loaded PLGA microparticles: Flow focusing versus double emulsion/solvent evaporation. J Microencapsul 28: 430-441, 2011.

14. Shive MS and Anderson JM: Biodegradation and biocompatibility of PLA and PLGA microspheres. Adv Drug Deliv Rev 28 5-24, 1997

15. Wang CW, Yang SP, Hu H, Du J and Li FH: Synthesis, characterization and in vitro and in vivo investigation of $\mathrm{C}_{3} \mathrm{~F}_{8}$-filled poly(lactic-co-glycolic acid) nanoparticles as an ultrasound contrast agent. Mol Med Rep 11: 1885-1890, 2015.

16. Cho SH, Kim JY and Kim JD: Dynamic surface tension of stable air-filled microbubbles prepared by freeze-drying a solution of lipid/surfactant mixture. Colloids and Surfaces A: Physicochemical and Engineering Aspects 284-285: 453-457, 2006.

17. Ma XY, Pan GM, Lu Z, Hu JS, Bei JZ, Jia JH and Wang SG: Preliminary study of oral polylactide microcapsulated insulin in vitro and in vivo. Diabetes Obes Metab 2: 243-250, 2000.

18. Kumari A, Yadav SK and Yadav SC: Biodegradable polymeric nanoparticles based drug delivery systems. Colloids Surf B Biointerfaces 75: 1-18, 2010.

19. Piñón-Segundo E, Nava-Arzaluz MG and Lechuga-Ballesteros D: Pharmaceutical polymeric nanoparticles prepared by the double emulsion-solvent evaporation technique. Recent Pat Drug Deliv Formul 6: 224-235, 2012.

20. Li XY, Yang ZL and Zhou HG: Studying the absorption spectrum properties of the gold nanosphere-The effect of the size on the absorption spectra of the anoparticles. J Zhangzhou Teachers Coll (Nat Sci) 17: 31-34, 2004.

21. Yuan F, Dellian M, Fukumura D, Leunig M, Berk DA, Torchilin VP and Jain RK: Vascular permeability in a human tumor xenograft: Molecular size dependence and cutoff size. Cancer Res 55: 3752-3756, 1995.

22. Koo OM, Rubinstein I and Onyuksel H: Role of nanotechnology in targeted drug delivery and imaging: A concise review. Nanomedicine 1: 193-212, 2005.

23. Panchapakesan B and Wickstrom E: Nanotechnology for sensing, imaging, and treating cancer. Surg Oncol Clin N Am 16 293-305, 2007. 